\title{
Discrete Spectrum of 2 + 1-Dimensional Nonlinear Schrödinger Equation and Dynamics of Lumps
}

\author{
Javier Villarroel, ${ }^{1}$ Julia Prada, ${ }^{1}$ and Pilar G. Estévez ${ }^{2}$ \\ ${ }^{1}$ Instituto Universitario de Física y Matemáticas, Facultad de Ciencias, Plaza Merced s/n, 37008 Salamanca, Spain \\ ${ }^{2}$ Departamento de Física Fundamental, Universidad de Salamanca, Facultad de Ciencias, Plaza Merced s/n, 37008 Salamanca, Spain
}

Correspondence should be addressed to Javier Villarroel; javier@usal.es

Received 15 June 2016; Accepted 29 August 2016

Academic Editor: Boris G. Konopelchenko

Copyright (C) 2016 Javier Villarroel et al. This is an open access article distributed under the Creative Commons Attribution License, which permits unrestricted use, distribution, and reproduction in any medium, provided the original work is properly cited.

\begin{abstract}
We consider a natural integrable generalization of nonlinear Schrödinger equation to $2+1$ dimensions. By studying the associated spectral operator we discover a rich discrete spectrum associated with regular rationally decaying solutions, the lumps, which display interesting nontrivial dynamics and scattering. Particular interest is placed in the dynamical evolution of the associated pulses. For all cases under study we find that the relevant dynamics corresponds to a central configuration of a certain $N$-body problem.
\end{abstract}

\section{The Physical System}

In this paper we consider the system of equations:

$$
\begin{array}{r}
i u_{t}+u_{x x}+2 u \partial_{x} n=0, \\
n_{y}+|u|^{2}=0,
\end{array}
$$

where $u(x, y, t)$ is a complex function, depending on three real variables $x, y, t$. We derive via the inverse scattering method several large classes of solutions that satisfy the boundary conditions $\left(r^{2} \equiv x^{2}+y^{2}\right)$,

$$
\begin{array}{r}
\lim _{r \rightarrow \infty}|u|(x, y, t)=1, \\
\lim _{r \rightarrow \infty} u_{x}=0, \\
n(x, y=-\infty, t)=0 .
\end{array}
$$

Note that in this case the system reads

$$
\begin{aligned}
& i u_{t}+u_{x x}+2 u \partial_{x} \int_{-\infty}^{y}\left(1-|u|^{2}\right) d y^{\prime}=0, \\
& n(x, y, t)=-y+\int_{-\infty}^{y}\left(1-|u|^{2}\left(x, y^{\prime}\right)\right) d y^{\prime} .
\end{aligned}
$$

Due to the overall simplicity, elegance, and potential physical interest, the above system is a natural interesting nonlinear $2+1$-dimensional integrable equation to study, even though it has not been related to any physical situation so far. To further motivate its study we note the following:

(1) The more general problem corresponding to the boundary conditions

$$
\begin{aligned}
\lim _{r \rightarrow \infty}|u|(x, y, t) & =C, \\
\lim _{r \rightarrow \infty} u_{x} & =n(x, y=-\infty, t)=0,
\end{aligned}
$$

where $C>0$ is a given nonnull constant is also solvable and reducible to problem (2) as follows. Let $\widehat{u}(x, y, t)$ be the unique solution to (1) under the boundary conditions (2). Then one proves easily that $u(x, y, t) \equiv C \widehat{u}\left(C x, C y, C^{2} t\right)$ also solves (1) and satisfies (4).

(2) System (3) arises as the compatibility of a Lax pair (see Section 2 below) and hence it is integrable. We show here that the operator has also a discrete spectrum, corresponding to potentials that solve (1) and (2): that is, it can be written as $u=1+\widetilde{u}$, where $\widetilde{u}$ is a regular, weakly decaying function. 
(3) If $u$ and $n$ only satisfy $|u|^{2} \in L_{1}\left(\mathbb{R}^{2}\right)$ and $\lim _{y \rightarrow-\infty} n(x, y)=0$, then (1) reads

$$
\begin{aligned}
i u_{t}+u_{x x}-2 u \partial_{x} \int_{-\infty}^{y} d y^{\prime}|u|^{2} & =0 \\
n(x, y, t) & =\int_{-\infty}^{y}|u|^{2}\left(x, y^{\prime}, t\right) d y^{\prime}
\end{aligned}
$$

This equation was first noticed to be integrable by Fokas [1] and also in Shul'man [2] who also embedded it into a general multiparameter family of equations. Note that in potentials satisfying $|u|^{2} \in L_{1}\left(\mathbb{R}^{2}\right)$ there is not discrete spectrum (see $[3,4]$ ).

(4) The further reduction to the manifold $x=y$ yields the nonlinear Schrödinger (NLS) equation. Hence (1) generalizes NLS to $2+1$ dimensions.

(5) The relationship between the previous problem and that corresponding to the boundary conditions (2) and

$\lambda n(x, y=-\infty, t)+(1-\lambda) n(x, y=\infty, t)=0$,

where $\lambda \in \mathbb{R}$ and $r^{2} \equiv x^{2}+y^{2}$, has been investigated in [5]. We note that the conserved quantities depend on the election of $\lambda$.

(6) There is an infinite number of conservation laws for this equation (see [5]), which includes, in particular, the functional

$$
M(t) \equiv \int\left(|u|^{2}(x, y)-1\right) d x d y .
$$

We note that the "amplitude" $|u|^{2}(x, y)-1$ of interesting solutions to (3) is naturally expected to be the physical observable but it is not definite positive; hence, the renormalized mass of the field $M \equiv M_{+}-$ $M_{-}$can take both signs.

(7) An interesting parabolic real version of (1) (i.e., letting $i u_{t} \rightarrow u_{t}$ ) is obtained as a particular reduction of the Self-Dual Yang Mills Equations; see [6].

Lump configurations (i.e., regular and rationally decaying solutions) are paradigmatic solutions of integrable equations in $2+1$ dimensions and as such they have been extensively studied in the last years. Their spectral interpretation was first unlocked in relation to KPI [7] (see also [8, 9]). A description of the KP equation, its physical origins, integrability, and soliton solutions can be found in [10]. Subsequently, lumps have been found in other integrable equations like DaveyStewartdon II (DSII) (see [11]) and the 2 + 1-Toda lattice (see [12-14]).

The dynamics of standard lumps on the plane is trivial: the motion is uniform; further, upon interaction, only a parallel shift on the asymptotic trajectory is to be found. Such uniform motion has been considered to rule the dynamics of localized pulses of integrable equations up until the late nineties. Remarkably it was found that KPI possess a new class of localized, real valued solutions that have a nontrivial asymptotic dynamics. Even though the simplest of these solutions has been known for a long time, the interesting scattering properties that they exhibit went unnoticed up until 1995 $[15,16]$. The spectral interpretation along with a derivation of the general class of those solutions was unlocked in [17, 18]. Nonstandard lumps were found to be associated with a new discrete spectrum of the time dependent Schrödinger operator corresponding to meromorphic eigenfunctions with poles of higher multiplicity and to what we term nonstandard pole divisors. (A comprehensive account of the spectrum of both KPI and KPII is given in [19].) The extension of these ideas and solutions to DSII equation via spectral analysis of the Dirac operator on the plane has been considered in [20]. We also note that KPI possesses, in addition, other localized, nondecaying solutions like line solitons $[21,22]$. The solution of the Cauchy problem in such a background is considered in $[14,23]$.

In this paper we show that the spectral operator that linearizes (3) possesses a rich discrete spectrum corresponding to smooth, rationally "decaying" lump configurations whose dynamical evolution and scattering are nontrivial. The physical behavior of these solutions is reminiscent to the aforementioned discrete spectrum and nonstandard lumps for KPI. We find that they are associated with higherorder pole meromorphic eigenfunctions of a similar discrete spectrum. Characterization of the discrete spectrum of this operator involves giving the pole multiplicity and an adequate pole divisor, which could be, in the spirit of the ideas of $[18,20]$, associated with integer winding numbers.

Direct methods to study this class of solutions have also been developed; see [24, 25] for KPI and [26] for DSII. See also [27] for related ideas. These direct algebraic methods, although they do not shed any light on the associated spectral problem, are quite powerful to undertake a general classification of the relevant class of solutions. In this regard note that in [28-31] Painleve's test for (3) was first considered and some special solutions, like line solitons, lumps, and dromion solutions, were found.

The organization of the paper is as follows. A convenient form of the linearizing Lax pair associated with (3) with boundary conditions (2) is introduced in Section 2, following the ideas of [32]. In Section 3 we study the discrete spectrum of meromorphic eigenfunctions. Different relations between the Laurent coefficients (LC) compatible with the Lax pair are established. In Section 4 we study several classes of lump solutions and their dynamics and physical properties. Particular interest is addressed to the problem of determining the number of solitons that ensue from given analytic structure and to determine the motion of these entities. We find that typically lump's dynamics is a superposition of the "center of mass" motion, which proceeds in a uniform way and individual, lump depending, motion that behaves as $|t|^{r}$ with $r<1$. Interestingly for all cases analyzed the dynamics corresponds to a central configuration of a certain $N$-body problem (a central configuration of the Euler-Lagrange $N$-body problem corresponds to interacting point masses wherein for each body the acceleration vector is directed towards the center of mass and proportional to the distance to the center of mass) where they fall to the mass center. Particularly interesting is the case, similar to the "homothetic Lagrange" solution in the 
tree body problem, when pulses are located at the vertexes of an equilateral triangle which collapses to the center along a straight line as $t^{1 / 3}$.

\section{Linear Problem}

As we have already pointed out system (3) arises as the compatibility of a pair of operators; see $[1,10,33,34]$ for genial ideas. Here we restrict our study to the class $u(x, y)$ of potentials that are nonsingular and satisfy (2). Under these boundary conditions a convenient form of the Lax pair, depending on a complex spectral parameter $k$, is given by the pair of operators:

$$
\begin{gathered}
L \mu \equiv \mu_{x y}+\frac{1}{k} \mu_{x}+\mu_{y}\left(k-\frac{u_{x}}{u}\right) \\
+\mu\left(1-\frac{1}{k} \frac{u_{x}}{u}-|u|^{2}\right)=0, \\
M \mu \equiv \mu_{x x}+i \mu_{t}+2 k \mu_{x}+2 m_{x} \mu=0 .
\end{gathered}
$$

We assume the existence of an eigenfunction normalized to 1 as $|k| \rightarrow \infty$. Then, (8) implies that $\mu(k)$ has the asymptotic expansion (with asymptotic coefficients $m_{1}, m_{2}, \ldots$ ):

$$
\begin{aligned}
\mu=1+\frac{m_{1}}{k}+\frac{m_{2}}{k^{2}}+\cdots, & \\
& |k| \longrightarrow \infty \text { where }|u|^{2}=1+\partial_{y} m_{1} .
\end{aligned}
$$

This expression permits recovering the physical amplitude $|u|^{2}$. To determine the phase of the potential $u$ consideration of the next order in the expansion is required. We find

$$
\frac{u_{x}}{u}=\frac{\left(\partial_{x y} m_{1}+\partial_{y} m_{2}-1 / 2 \partial_{y}\left(m_{1}\right)^{2}\right)}{\left(1+\partial_{y} m_{1}\right)}
$$

Thus, the potential is determined from

$$
\begin{aligned}
|u|^{2} & =1+\partial_{y} m_{1} \\
i \partial_{x} \arg u & =\frac{1}{2} \partial_{y} \frac{\left(\partial_{x} m_{1}-m_{1}^{2}+2 m_{2}\right)}{\left(1+\partial_{y} m_{1}\right)} .
\end{aligned}
$$

\section{Discrete Spectrum and Relationships among Laurent Coefficients}

Basic Assumption. The basic assumption of this work is the existence of a solution to (8) and (9), which has a finite or denumerable number of singularities $\left\{k_{n}, n=1, \ldots, \infty\right\}$. We suppose that all singularities are higher-order poles and that $\mu(k)$ is analytic away from the singularities. Thus, around any pole $k=k_{1}, \mu(k)$ must have a local Laurent expansion:

$$
\mu(k)=\mu_{\text {sing. }}^{\left.k_{1}\right)}(k)+\mu_{\text {reg. }}^{\left.k_{1}\right)}(k),
$$

where

$$
\begin{aligned}
& \mu_{\text {reg. }}^{\left.k_{1}\right)}(k) \equiv \sum_{r=0}^{\infty} v_{r}\left(k-k_{1}\right)^{r}, \\
& \mu_{\text {sing. }}^{\left.k_{1}\right)}(k) \equiv \sum_{r=1}^{m} \frac{\phi_{r}}{\left(k-k_{1}\right)^{r}},
\end{aligned}
$$

are, respectively, the regular and singular parts of the eigenfunction at the pole and $\phi_{r}=\phi_{r}(x, y, t), \nu_{r}=v_{r}(x, y, t)$ are the Laurent coefficients (LC). Notice that $\mu_{\text {sing. }}^{\left.k_{1}\right)}(k)$ is also termed the singularity principal part or the pole divisor.

Unlike what happens in the regular case, where the eigenfunction is fixed by the corresponding $\bar{\partial}$-problem, when singularities exist the inverse problem does not fix uniquely the singular part. Additional information relating different coefficients of the poles divisor is required. In this section we consider examples of meromorphic eigenfunctions related to nonsingular potentials and determine different relationships between coefficients of the poles divisor. We posit the existence of a linear relationship between coefficients of the Laurent expansion in the form

$$
v_{r}=\sum_{j=-m}^{r-1} f_{j}(x, y, t) v_{j} \quad \text { for a certain } r \geq 0,
$$

where $m$ is the pole multiplicity and $f_{j}$ are certain functions to be determined. We say that the integer $1+r$ is the index.

3.1. Simple Poles. We shall first suppose that $\mu \equiv \mu(x, y, t, \cdot)$ is an eigenfunction of (8), (9) with a meromorphic dependence on $k$ and assume that $k_{1}$ is a simple pole of $\mu$. Let $\phi_{1, k_{1}} \equiv \phi$ be the residue of $\mu(k)$ at $k_{1}$. Equation (14) reads

$$
\mu(x, y, t, k)=\frac{\phi(x, y, t)}{k-k_{1}}+\mu_{\text {reg. }}^{\left.k_{1}\right)}(x, y, t, k) .
$$

Indeed, letting $k \rightarrow k_{1}$ in (8), (9) we obtain, at dominant orders, that $\phi$ and $\nu \equiv v_{0}$ must satisfy the system of equations:

$$
\begin{aligned}
& \phi_{x y}+\frac{1}{k_{1}} \phi_{x}+\phi_{y}\left(k_{1}-\frac{u_{x}}{u}\right) \\
& +\phi\left(1-\frac{1}{k_{1}} \frac{u_{x}}{u}-|u|^{2}\right)=0, \\
& v_{x y}+\frac{1}{k_{1}} v_{x}+v_{y}\left(k_{1}-\frac{u_{x}}{u}\right)+v\left(1-\frac{1}{k_{1}} \frac{u_{x}}{u}-|u|^{2}\right) \\
& \quad+\left(\phi_{y}-\frac{1}{k_{1}^{2}} \phi_{x}+\frac{1}{k_{1}^{2}} \frac{u_{x}}{u} \phi\right)=0, \\
& \phi_{x x}+i \phi_{t}+2 k_{1} \phi_{x}+2 m_{x} \phi=0, \\
& v_{x x}+i v_{t}+2 k_{1} v_{x}+2 m_{x} \nu+2 \phi_{x}=0 .
\end{aligned}
$$

3.1.1. Simple Poles of Index One. We first consider the simplest linear relationship when

$$
\begin{aligned}
& r=0, \\
& v=f \phi \quad \text { for certain } f(x, y, t) .
\end{aligned}
$$


Then, from the previous equations $f(x, y, t)$ must satisfy

$$
\begin{aligned}
& \left(f_{x y}+\frac{f_{x}}{k_{1}}+k_{1} f_{y}-\left(f_{y}-\frac{1}{k_{1}^{2}}\right) \frac{u_{x}}{u}\right) \phi+\phi_{y}\left(f_{x}+1\right) \\
& \quad+\phi_{x}\left(f_{y}-\frac{1}{k_{1}^{2}}\right)=0, \\
& \phi\left(f_{x x}+i f_{t}+2 k_{1} f_{x}\right)+2 \phi_{x}\left(f_{x}+1\right)=0 .
\end{aligned}
$$

We can satisfy these equations by requiring $f$ to solve the following system of constant differential equations:

$$
f_{y}-\frac{1}{k_{1}^{2}}=f_{x}+1=f_{x x}+i f_{t}+2 k_{1} f_{x}=0 .
$$

It follows that

$$
f=-x-2 i k_{1} t+\frac{y}{k_{1}^{2}}+\gamma, \quad \text { where } \gamma \equiv \gamma_{R}+i \gamma_{I} \in \mathbb{C} .
$$

Therefore, eigenfunctions of (8) with the structure (16) can be constructed by requiring that $\phi$ solves (17) and that the relationship

$$
\nu_{0}=\left(-x-2 i k_{1} t+\frac{y}{k_{1}^{2}}+\gamma\right) \phi
$$

holds for some complex constant $\gamma$. A similar relation was first established for KPI in [7]. Whenever such a situation holds we say that the pole divisor is standard. We next study the possibility of having nonstandard pole divisors.

3.1.2. Simple Poles of Index 2. We now show that the analytic structure (16) for eigenfunctions of (8) does not necessarily imply that (24) holds and different linear relationship between the LCs may hold; concretely we consider here the case when $v_{1}, v_{0}, v_{-1} \equiv \phi$ are related as

$$
\begin{aligned}
r & =1, \\
v_{1} & =f v_{0}+g \phi \text { for certain } f(x, y, t), g(x, y, t) .
\end{aligned}
$$

In this case we also need to supplement (17), (18) with higherorder equations:

$$
\begin{aligned}
& \left(\partial_{x x}+i \partial_{t}+2 k \partial_{x}+2 m_{x}\right) \nu_{1}+2 \partial_{x} \nu_{0}=0 \\
& v_{1 x y}+\frac{1}{k_{1}} v_{1 x}+\left(k_{1}-\frac{u_{x}}{u}\right) v_{1 y} \\
& \quad+\left(1-\frac{1}{k_{1}} \frac{u_{x}}{u}-|u|^{2}\right) v_{1}-\frac{v_{0 x}}{k_{1}^{2}}+v_{0 y}+\frac{u_{x}}{k_{1}^{2} u} v_{0}=0
\end{aligned}
$$

By insertion of (25) into (26) we obtain

$$
\begin{gathered}
f_{x x} v_{0}+g_{x x} \phi+2 f_{x} \nu_{0 x}+2 g_{x} \phi_{x}+i\left(f_{t} \nu_{0}+g_{t} \phi\right) \\
+2 k_{1}\left(f_{x} v_{0}+g_{x} \phi\right)+2 v_{0 x}-2 f \phi_{x}=0 .
\end{gathered}
$$

This equation will be satisfied if $f$ is again given by (23) and $g$ solves

$$
g_{x x}+i g_{t}+2 k_{1} g_{x}=0, \quad g_{x}=f
$$

By integration

$$
\begin{aligned}
v_{1}= & f \nu_{0}+g \phi \\
\equiv & \left(-x-2 i k_{1} t+\frac{y}{k_{1}^{2}}+\gamma\right) \nu_{0} \\
& \quad-\left(\frac{f^{2}}{2}+i t+\frac{y}{k_{1}^{3}}-\delta\right) \phi,
\end{aligned}
$$

where $\gamma$ and $\delta$ are complex constants. Equation (30) gives yet another relationship between the three first LCs $\phi, v_{0}$, and $\nu_{1}$ of simple pole eigenfunctions compatible with the Lax pair.

3.2. Poles of Order Two. We now show how the above ideas can be extended to cover the case when the eigenfunction has a pole of multiplicity 2 at some point $k=k_{1}$ : that is, $m=2$ in (14) and $\mu_{\text {sing. }}^{\left.k_{1}\right)}(k) \equiv \psi /\left(k-k_{1}\right)^{2}+\phi /\left(k-k_{1}\right)$, where, to avoid an awkward notation, we write $\phi_{2, k_{1}} \equiv \psi, \phi_{1, k_{1}} \equiv \phi$ and also $v_{0, k_{1}} \equiv \nu$. By letting $k \rightarrow k_{1}$ in (8) we find that the main coefficients must satisfy $\psi_{x x}+i \psi_{t}+2 k_{1} \psi_{x}+2 m_{x} \psi=0$, and also

$$
\begin{aligned}
\psi_{x y} & +\frac{\psi_{x}}{k_{1}}+\left(k_{1}-\frac{u_{x}}{u}\right) \psi_{y} \\
& +\left(1-\frac{1}{k_{1}} \frac{u_{x}}{u}-|u|^{2}\right) \psi=0, \\
\phi_{x y}+\frac{1}{k_{1}} \phi_{x}+\left(k_{1}-\frac{u_{x}}{u}\right) \phi_{y}+\left(1-\frac{1}{k_{1}} \frac{u_{x}}{u}-|u|^{2}\right) \phi & \\
& +\psi_{y}-\frac{1}{k_{1}^{2}} \psi_{x}+\frac{1}{k_{1}^{2}} \frac{u_{x}}{u} \psi=0, \\
v_{x y} & +\frac{1}{k_{1}} v_{x}+\left(k_{1}-\frac{u_{x}}{u}\right) v_{y}+\left(1-\frac{1}{k_{1}} \frac{u_{x}}{u}-|u|^{2}\right) \nu \\
& -\frac{1}{k_{1}^{2}} \phi_{x}+\phi_{y}+\frac{1}{k_{1}^{2}} \frac{u_{x}}{u} \phi+\frac{1}{k_{1}^{3}} \psi_{x}-\frac{1}{k_{1}^{3}} \frac{u_{x}}{u} \psi=0, \\
\phi_{x x} & +i \phi_{t}+2 k_{1} \phi_{x}+2 m_{x} \phi+2 \psi_{x}=0, \\
v_{x x}+i v_{t} & +2 k_{1} v_{x}+2 m_{x} v+2 \phi_{x}=0 .
\end{aligned}
$$

Again, the assumed analytic structure does not fix uniquely the way LCs are related. We next consider several possibilities.

3.2.1. Poles of Order Two and Index Two. We first assume that the span $\left\langle\psi, \phi, v_{0}\right\rangle$ of all linear combinations of $\psi, \phi$ and $\nu_{0} \equiv$ $\nu$ with coefficients dependent on $x, y, t$ is generated by just one of them; concretely we require

$$
\begin{aligned}
\operatorname{Dim} .\langle\psi, \phi, \nu\rangle & =1 ; \\
\text { alternatively } \phi & =f \psi, \\
\nu & =h \psi,
\end{aligned}
$$


where $f, h$ depend on $x, y, t$. As previously mentioned, from the $\phi$-equations we find $f$ to be given by (23), while the remaining equations yield that $h$ must satisfy

$$
h_{x x}+i h_{t}+2 k_{1} h_{x}+2 f_{x}=0, \quad h_{x}+f=0
$$

or, alternatively, $h_{f}-f=0, i h_{t}=1$. It follows that (32) is compatible with the given analytic structure provided $f$ is given by (23) and for some complex constants $\gamma, \delta$

$$
h=\frac{f^{2}}{2}-i t-\frac{y}{k_{1}^{3}}+\delta
$$

3.2.2. Poles of Order Two and Index Three. A different possibility obtains by requiring now

$$
\operatorname{Dim} .\langle\psi, \phi\rangle=1 \text {, }
$$

$\operatorname{Dim} .\left\langle\psi, \phi, v_{0}, v_{1}\right\rangle=2$,

$$
\begin{aligned}
\text { or } \phi & =f \psi, \\
v_{1} & =A(x, y, t) \nu_{0}+B(x, y, t) \psi .
\end{aligned}
$$

In this case, letting $k \rightarrow k_{1}$ in (8) we obtain that

$$
\begin{aligned}
& \psi_{x x}+i \psi_{t}+2 k_{1} \psi_{x}+2 m_{x} \psi=0, \\
& \psi_{x y}+\frac{\psi_{x}}{k_{1}}+\left(k_{1}-\frac{u_{x}}{u}\right) \psi_{y} \\
& +\left(1-\frac{1}{k_{1}} \frac{u_{x}}{u}-|u|^{2}\right) \psi=0, \\
& v_{1 x y}+\frac{v_{1 x}}{k_{1}}+\left(k_{1}-\frac{u_{x}}{u}\right) \nu_{1 y}+\left(1-\frac{1}{k_{1}} \frac{u_{x}}{u}-|u|^{2}\right) \nu_{1} \\
& \quad-\frac{1}{k_{1}^{2}} v_{0 x}+v_{0 y}+\frac{1}{k_{1}^{2}} \frac{u_{x}}{u} v_{0}+\frac{1}{k_{1}^{3}} \phi_{x}-\frac{1}{k_{1}^{3}} \frac{u_{x}}{u} \phi \\
& \quad-\frac{1}{k_{1}^{4}} \psi_{x}+\frac{1}{k_{1}^{4}} \frac{u_{x}}{u} \psi=0, \\
& \left(\partial_{x x}+i \partial_{t}+2 k_{1} \partial_{x}+2 m_{x}\right) \nu_{1}+2 \partial_{x} \nu_{0}=0 .
\end{aligned}
$$

Hence, in view of (36)

$$
\begin{aligned}
A_{x x} \nu_{0} & +B_{x x} \psi+2 A_{x} v_{0 x}+2 B_{x} \psi_{x}+i\left(A_{t} \nu_{0}+B_{t} \psi\right) \\
& +2 k_{1}\left(A_{x} v_{0}+B_{x} \psi\right)+2 v_{0 x}-2 A\left(f \psi_{x}-\psi\right) \\
= & 0 .
\end{aligned}
$$

Requiring independence of $\nu_{0}, \psi$ we obtain that $A, B$ must solve

$$
\begin{aligned}
A_{x x}+i A_{t}+2 k_{1} A_{x} & =A_{x}+1=0, \\
B_{x x}+i B_{t}+2 k_{1} B_{x}+2 A & =B_{x}-A f=0 .
\end{aligned}
$$

Hence, we obtain that $A=f+\eta$ and $B=(-1 / 3)\left(f^{3}+\right.$ $\left.(3 / 2) \eta f^{2}\right)+i \eta t+r(y)$ where $\eta$ is a complex constant and $r(y)$ an arbitrary function of $y$. The $y$-equation gives

$$
\begin{aligned}
-B_{y}-\frac{1}{k_{1}^{3}} f+\frac{1}{k_{1}^{4}}-\frac{A}{k_{1}^{2}} f+\frac{A}{k_{1}^{3}} & =0 \\
\operatorname{or} r(y) & =\left(\frac{\eta}{k_{1}^{3}}+\frac{1}{k_{1}^{4}}\right) y+r_{0} .
\end{aligned}
$$

Thus, (36) is satisfied provided $f$ is given by (23) and

$$
\begin{aligned}
& A=(f+\eta), \\
& B=\frac{-1}{3}\left(f^{3}+\frac{3}{2 \eta f^{2}}\right)+i \eta t+\left(\frac{\eta}{k_{1}^{3}}+\frac{1}{k_{1}^{4}}\right) y+r_{0} .
\end{aligned}
$$

\section{Determination of Classes of Potentials}

In this section we determine several classes solutions of (1) by considering proper election of meromorphic eigenfunctions and relationships between LCs of the form (15).

4.1. Potentials Corresponding to Simple Poles of Index One. Suppose first that $\mu(k)$ is a meromorphic eigenfunction that has the representation (14) with a finite even number $2 N<\infty$ of simple poles. We make the following assumptions (denoted as condition $\mathrm{Cl}$ ):

(1) Poles come in pairs $k_{j}, k_{j+N}$ with corresponding residues $\phi_{j}, \phi_{\bar{j}}$ :

$$
\mu(k)=1+\sum_{j=1}^{N}\left(\frac{\phi_{j}}{k-k_{j}}+\frac{\phi_{\bar{j}}}{k+\bar{k}_{j}}\right) .
$$

(2) At every pole $k_{\alpha}, \alpha=1, \ldots, 2 N$ the pole divisor is standard and hence satisfies (24):

(3)

$$
\begin{gathered}
k_{j R} \neq 0, \\
k_{j+N}=-\bar{k}_{j}, \\
\gamma_{j+N}=\bar{\gamma}_{j},
\end{gathered}
$$

Suppose that $\mu$ has just two poles $k_{1},-\bar{k}_{1}$. In this case from the previous assumptions and (24) we must have

$$
\begin{gathered}
v_{0}\left(k_{1}\right) \equiv 1+\frac{\phi_{\overline{1}}}{k_{1}+\bar{k}_{1}}=f_{1} \phi_{1}, \\
v_{0}\left(-\bar{k}_{1}\right) \equiv 1-\frac{\phi_{1}}{\bar{k}_{1}+k_{1}}=\bar{f}_{1} \phi_{\overline{1}} .
\end{gathered}
$$

Solving this system of linear equations and using (10) we find the potential

$$
|u|^{2}=1+\partial_{y}\left(\phi_{1}+\phi_{\overline{1}}\right)=1-\partial_{y x} \log \Delta
$$

while $n=-y+\partial_{x} \log \Delta$, 
where $\Delta$ is the determinant of the associated matrix:

$$
\Delta \equiv f_{1} \bar{f}_{1}+\frac{1}{\left(k_{1}+\bar{k}_{1}\right)^{2}} \text {. }
$$

The field $u$ given by (46) will be called the basic lump solution of (3). Obviously, if $k_{1} \equiv k_{1 R}+i k_{1 I} \equiv a+i b$, where $a \equiv k_{1 R} \neq 0$, the solution is regular on the entire plane and decreases rationally.

Consider a new Galilean frame $\left(x^{\prime}, y^{\prime}\right)$ moving with velocities $\left(c,-b c^{2}\right)$ :

$$
\begin{aligned}
& x^{\prime}=x-c t-\tilde{\gamma}, \\
& y^{\prime}=y+b c^{2} t+\frac{\gamma_{0 I}}{\rho}, \\
& c=\frac{a^{2}+b^{2}}{b}, \\
& \beta=\frac{a^{2}-b^{2}}{\left(a^{2}+b^{2}\right)^{2}}, \\
& \rho=-\frac{2 a b}{\left(a^{2}+b^{2}\right)^{2}}, \\
& \tilde{\gamma} \equiv \gamma_{0 R}-\frac{a^{2}-b^{2}}{2 a b} \gamma_{0 I} .
\end{aligned}
$$

One finds also convenient to go to a frame of skew coordinates $z, y^{\prime}$ where $x^{\prime}$, and $y^{\prime}$ are defined in (48) and $z \equiv x^{\prime}-\beta y^{\prime}$ is a shear transformation along the $x$-axis, where $\beta$ represents a slanting coefficient. In this frame the potential reads

$$
\begin{aligned}
v \equiv|u|^{2}-1 \\
=-2 \beta\left(z^{2}+\frac{\rho^{2} y^{\prime}}{\beta}\left(\beta y^{\prime}-2 x^{\prime}\right)-\frac{1}{4 a^{2}}\right) \frac{1}{\Delta^{2}}, \\
\text { where } \Delta=z^{2}+\rho^{2} y^{\prime 2}+\frac{1}{4 a^{2}} .
\end{aligned}
$$

Thus, in this inertial frame the solution is at rest for all time (objects moving uniformly with velocities $\left(c,-b c^{2}\right)$ in the unprimed frame remain at rest in the transformed primed frame) and strongly localized. However, the maxima structure is richer than what might have been expected. Inspection shows that critical points solve $x^{\prime}=\sigma y^{\prime} /\left(a^{2}+b^{2}\right)$, $\sigma= \pm 1$. With $\sigma=1$ we find that critical points are $\left(x^{\prime}, y^{\prime}\right)=$ $(0,0)$ and

$$
\begin{aligned}
& x^{\prime}= \pm \frac{1}{4 a b} \sqrt{3 b^{2}-a^{2}}, \\
& y^{\prime}= \pm \frac{a^{2}+b^{2}}{4 a b} \sqrt{3 b^{2}-a^{2}}
\end{aligned}
$$

while for $\sigma=-1$ one has

$$
\begin{aligned}
& y^{\prime}= \pm \frac{a^{2}+b^{2}}{4 a^{2}} \sqrt{3 a^{2}-b^{2}}, \\
& x^{\prime}=\mp \frac{1}{4 a^{2}} \sqrt{3 a^{2}-b^{2}} .
\end{aligned}
$$

These points are all candidates to maxima and minima; the number of them will vary depending on the values of the parameters. The parameter space is a two-dimensional plane deprived of the straight line $a=0$. To describe the situation in the general case we restrict, with no loss of generality, to the first quadrant on the parameter space. The situation varies according to which of the regions

$$
\begin{aligned}
& C_{1} \equiv\left\{(a, b): a \leq \frac{b}{\sqrt{3}}\right\}, \\
& C_{2} \equiv\left\{(a, b): \frac{b}{\sqrt{3}} \leq a \leq \sqrt{3} b\right\}, \\
& C_{3} \equiv\{(a, b): 0 \leq \sqrt{3} b \leq a\}
\end{aligned}
$$

do parameters belong. If $(a, b)$ is in the interior of $C_{2}$ then two of the four points described above are maxima and the other two are minima. Point $\left(x^{\prime}, y^{\prime}\right)=(0,0)$ is a saddle point between the formers at which $|u|=\left|3 a^{2}-b^{2}\right| /\left(a^{2}+b^{2}\right)$.

Particular cases are $b=0$ (previously studied) and $a=b$. In this case

$$
v \equiv|u|^{2}-1=\frac{x^{\prime} y^{\prime}}{a^{4}\left(x^{\prime 2}+y^{\prime 2} / 4 a^{4}+1 / 4 a^{2}\right)^{2}} .
$$

It has two symmetric maxima located at $p= \pm \sqrt{2}(1 / 4 a, a / 2)$ at which $v \equiv|u|^{2}-1=1$ while minima are to be found at the mirror images points at which $v=-1$.

When parameters satisfy $a=\sqrt{3} b$ we obtain a configuration having a maximum $\left(x^{\prime}, y^{\prime}\right)=(0,0)$ at which $v=3$; the minimum lump's amplitude $v=-1$ is to be found at the points $\left(x^{\prime}, y^{\prime}\right)= \pm(\sqrt{8} / 12 b)\left(1,-4 b^{2}\right)$. At the other boundary point $b=\sqrt{3} a>0$ the situation is opposite and is rather reminiscent of dark solitons; it corresponds to a configuration having minimum amplitude $v=-1$ at the origin and maximum amplitude $v=3 / 25$ at the points $\left(x^{\prime}, y^{\prime}\right)=$ $\pm(\sqrt{8} / 4 \sqrt{3} a)\left(1,4 a^{2}\right)$. Thus, generically the configuration is multipeaked, localized on an entire region containing several maxima.

4.2. Potentials Corresponding to Index Two. Here we investigate potentials associated with meromorphic eigenfunctions satisfying the following conditions (C2):

(1) Poles come in pairs $k_{j}, k_{j+N}, j=1, \ldots, N$ with $k_{j+N}=$ $-\bar{k}_{j}$ :

$$
\mu(k)=1+\sum_{j=1}^{N}\left(\frac{\phi_{j}}{k-k_{j}}+\frac{\phi_{\bar{j}}}{k+\bar{k}_{j}}+\frac{\psi_{\bar{j}}}{\left(k+\bar{k}_{j}\right)^{2}}\right) .
$$

(2) For $j=1, \ldots, N$ the poles $k_{j}$ are simple with index 2 (i.e., they satisfy (30)). Besides $k_{j}, j=N+1, \ldots, 2 N$, are double poles with index 2 in the sense of (32), (34) with constants satisfying $k_{j R} \neq 0, \gamma_{\bar{j}}=\bar{\gamma}_{j}, \delta_{\bar{j}}=-\bar{\delta}_{j}$.

We suppose that $\mu$ has just two poles $k_{1},-\bar{k}_{1}$ with the above properties. In this case one can prove that

$$
|u|^{2}=1+\partial_{y}\left(\phi_{1}+\phi_{\overline{1}}\right)=1-\partial_{y x} \log \Delta,
$$


where $\Delta$ is the following definite-positive polynomial:

$$
\begin{aligned}
\Delta \equiv\left|h_{1}\right|^{2}+\left|f_{1}+\frac{1}{2 a}\right|^{2} \frac{1}{4 a^{2}} & +\frac{1}{16 a^{4}}, \\
h_{1} & =-\left(\frac{f_{1}^{2}}{2}+i t+\frac{y}{k_{1}^{3}}-\delta\right), \\
f_{1} & =-x-2 i k_{1} t+\frac{y}{k_{1}^{2}}+\gamma .
\end{aligned}
$$

Note how it depends on 3 complex parameters $k_{1}, \gamma_{1}, \delta$.

We next study the dynamical properties of the field $u$ of (56), (57). Clearly $|u|^{2}$ is a rational function, regular on the entire plane which decreases as $1 / r^{2}$ as $r^{2} \equiv x^{2}+y^{2} \rightarrow \infty$. Again we find it convenient to use coordinates defined by (48) (see also (49)) in terms of which the " $\tau$ "-function reads (we drop some irrelevant parameters)

$$
\Delta=h_{R}^{2}+h_{I}^{2}+\frac{1}{4 a^{2}}\left[\left(z+\frac{1}{2 a}\right)^{2}+\rho^{2} y^{\prime 2}\right]+\frac{1}{16 a^{4}} .
$$

Note also that

$$
\begin{aligned}
& h_{R}=\frac{1}{2} z^{2}-\frac{\rho^{2} y^{\prime 2}}{2}+\varepsilon\left(\frac{b y^{\prime}}{\left(a^{2}+b^{2}\right)^{2}}-t\right), \\
& h_{I}=-\rho z y^{\prime}+\frac{b\left(3 a^{2}-b^{2}\right)}{\left(a^{2}+b^{2}\right)^{3}} y^{\prime}-\frac{4 a^{2}}{a^{2}+b^{2}} t,
\end{aligned}
$$

where $z \equiv x^{\prime}-\beta y^{\prime}, \varepsilon=\left(\left(a^{2}-3 b^{2}\right) /\left(a^{2}+b^{2}\right)\right)(a / b), k_{1} \equiv a+i b$, $h_{\overline{1}}=h_{R}+i h_{I}$.

Inspection of the solution shows that it corresponds to a coherent two pulsed structures. Each of the pulses has a rich internal structure with several maxima and minima in a similar disposition to that corresponding to the standard lump.

The dynamical behavior of this configuration is different and far more subtle than previously found. It turns out that lumps are not at rest in any inertial frame.

As indicated we consider a system of skew coordinates $z$, $y^{\prime}$ defined in (48). We claim that the solutions to

$$
\begin{aligned}
\Delta\left(z^{ \pm \infty}, y^{\prime \pm \infty}, t\right)= & O(t), \\
& \text { where }\left(z^{ \pm \infty}(t), y^{\prime \pm \infty}(t)\right) \equiv p^{ \pm \infty}
\end{aligned}
$$

give the pulse's positions as $t \rightarrow \pm \infty$. Indeed,

$$
\begin{aligned}
\partial_{y} \Delta, \partial_{z} \Delta\left(z^{ \pm \infty}, y^{\prime \pm \infty}\right) & =O(t), \\
u\left(z^{ \pm \infty}, y^{ \pm \infty}, t\right) & =O(1) .
\end{aligned}
$$

Note that $-p^{ \pm \infty}$ solves (60) if $p^{ \pm \infty}$ does; thus, the second pulse is the mirror image of the first respect to the origin, and it suffices to describe the motion of the first of them.

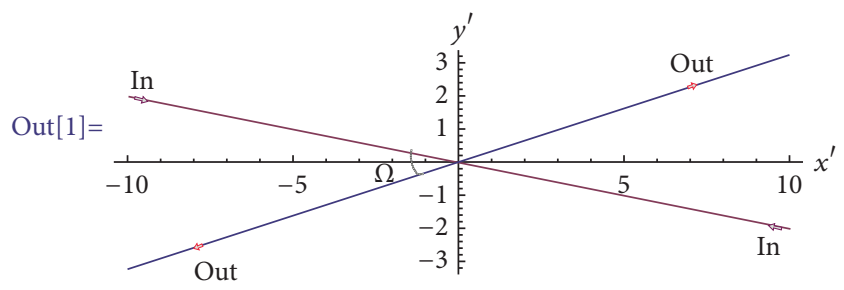

FIGURE 1: The path of the two pulses for the cases incoming $(t=-\infty)$ and outgoing $(t=\infty)$ corresponding to $a=0.3$ with the scattering angle indicated.

We first consider the case $a=b$ for which the dynamics and scattering process are easier to understand. In this case the moving frame (48) and solution are given by

$$
\begin{aligned}
x^{\prime}= & x-2 a t-\gamma_{R}, \\
y^{\prime}= & y+4 a^{3} t+2 a^{2} \gamma_{0 I}, \\
\rho= & -\frac{1}{2 a^{2}}, \\
\Delta= & \left(\frac{x^{\prime 2}}{2}-\frac{y^{\prime 2}}{8 a^{4}}-\frac{y^{\prime}}{4 a^{3}}+t\right)^{2} \\
& +\left(\frac{x^{\prime} y^{\prime}}{2 a^{2}}+\frac{y^{\prime}}{4 a^{3}}-2 t\right)^{2} \\
& +\frac{1}{4 a^{2}}\left[\left(x^{\prime}+\frac{1}{2 a}\right)^{2}+\frac{y^{\prime 2}}{4 a^{4}}+\frac{1}{4 a^{2}}\right] .
\end{aligned}
$$

We then find from (60) that asymptotically the first of the pulses has coordinates $p^{ \pm \infty}(t) \equiv\left(x^{\prime \pm \infty}(t), y^{\prime \pm \infty}(t)\right)$, where $\zeta^{ \pm}=(\sqrt{5} \mp 1)^{1 / 2}, \iota^{ \pm}= \pm 4 a^{2} / \zeta^{ \pm}$, and

$$
\begin{aligned}
x^{\prime \pm \infty} & =\zeta^{ \pm} \sqrt{|t|}+x_{0}^{ \pm \infty}+O\left(|t|^{-1 / 2}\right), \\
y^{\prime \pm} & =\iota^{ \pm} \sqrt{|t|}+y_{0}^{ \pm}+O\left(|t|^{-1 / 2}\right) .
\end{aligned}
$$

Thus, in the moving reference frame the trajectories as $t \rightarrow \pm \infty$ are straight lines with different slopes. Notice that initially $(t \rightarrow-\infty)$ pulses are located in the second and fourth quadrants and will move to the first and third ones as $t \rightarrow \infty$. For moderate times they collide head-on henceforth undergoing a scattering process. In Figures 1 and 2 we show the collision path and form of the pulses in solution (57) before and after scattering. The scattering angle $\Omega$ is easily found to be given by

$$
\cos \Omega=\frac{1-4 a^{4}}{\sqrt{\left(1+4 a^{4}\right)^{2}+4 a^{4}}} .
$$

Note that $\cos \Omega$ attains a maximum value 1 if (formally) $a=$ 0 and decreases towards $\cos \Omega=-1$ as $a \rightarrow \infty$. Thus, transparent scattering is obtained when $a=b=0$ while perpendicular scattering corresponds to lumps with $a^{2}=1 / 2$.

In the rest at frame the motion is the composition of a uniform motion $\left(2 a t,-4 a^{3} t\right)$ and the slower one given 


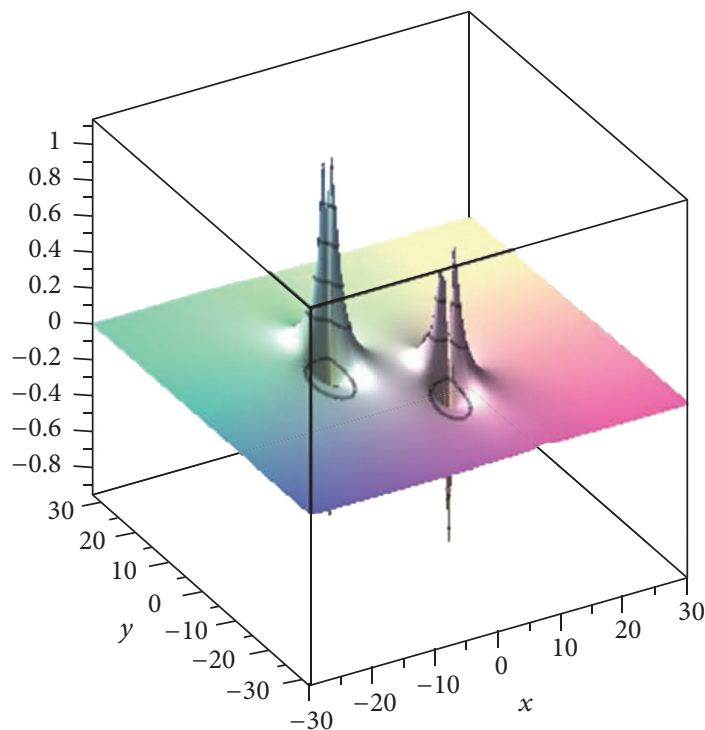

(a)

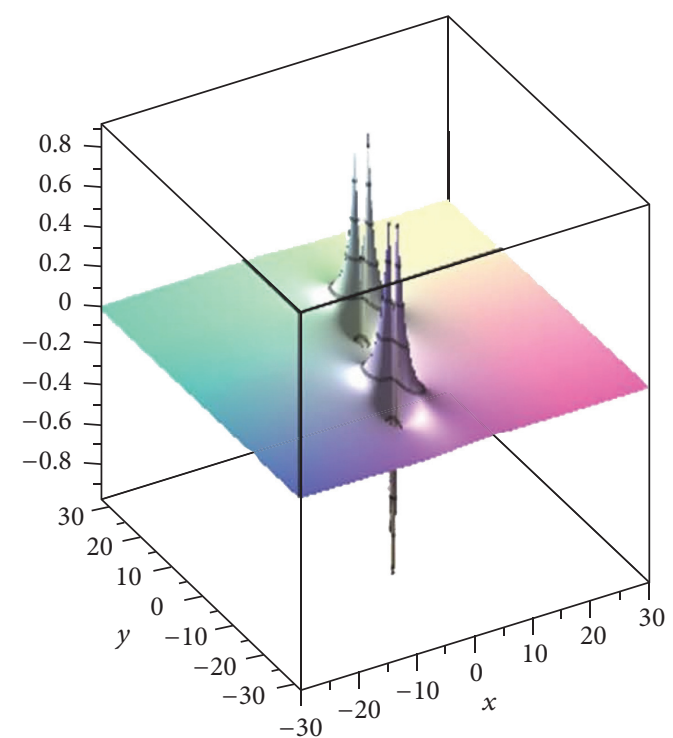

(b)

FIGURE 2: Location and form of the two pulses: incoming $(t=-10)$ and outgoing $(t=10)$ corresponding to the election of $a=b=1$. The scattering process is clear.

by (63). Indeed when $a=b$ both coordinate frames are related by a pure Galilean transformation and objects at rest in the primed frame pick a uniform motion with velocities $\left(2 a,-4 a^{3}\right)$ in the original frame. Hence, in the stationary frame the path of the second pulse is no longer the mirror image of the first.

4.3. Potentials Corresponding to Double Poles with Index Three. We now study potentials that correspond to eigenfunctions with double poles with index three. Concretely, assume the following:

(1) Poles come in pairs $k_{j}, k_{j+N}, j=1, \ldots, N$ with $k_{j+N}=$ $-\bar{k}_{j} \equiv k_{\bar{j}}$. Hence,

$$
\begin{aligned}
& \mu(k)=1+\sum_{j=1}^{N}\left(\frac{\phi_{j}}{k-k_{j}}+\frac{\psi_{j}}{\left(k-k_{j}\right)^{2}}+\frac{\phi_{\bar{j}}}{k+\bar{k}_{j}}\right. \\
& \left.+\frac{\psi_{\bar{j}}}{\left(k+\bar{k}_{j}\right)^{2}}\right) .
\end{aligned}
$$

(2) The multiplicity of every pole is two and the index three; namely, all pole divisors satisfy (36).

We consider the simplest such potential when $N=1$.

4.3.1. Pulses of Index Three. We consider in detail the physical properties and dynamics in the case $N=1$. The potential is given by formula (46) where the tau function is

$$
\Delta=\left|B\left(k_{1}\right)\right|^{2}+\frac{4}{(2 a)^{6}}+\frac{\left|f\left(k_{1}\right)\right|^{4}}{(2 a)^{2}}+4 \frac{\left(\operatorname{Im} f\left(k_{1}\right)\right)^{2}}{(2 a)^{4}}
$$

$\geq 0$.
Here $k_{1} \equiv a+i b$ and $\eta, \delta$ are complex constants, $v, \rho$ are defined in (49), and

$$
C \equiv-i \eta+b v^{2} \omega, \quad \omega=\frac{1}{k_{1}^{3}}\left(\frac{1}{k_{1}}+\eta\right) .
$$

The position and dynamics of the associated pulses are determined mainly by the constant $\eta$ (alternatively by $C \equiv$ $C_{R}+i C_{I}$ ). Concretely, we have the following.

Proposition 1. (1) If the constant $C=0$ the solution is a multipeaked traveling wave of solitonic nature (i.e., stationary in the frame moving with the soliton).

(2) When $C \neq 0$ the solution is nonstationary. There are exactly three traveling pulses describing "homothetic motion." Namely, the motion in the frame at rest is a superposition of a "center of mass" uniform motion and an individual and slower motion proportional to $t^{1 / 3}$.

(3) In the "center of mass frame" pulses are located at the vertex of a triangle with center of gravity at the origin (see Figure 3 where this situation is shown). The time evolution contracts but does not deform the whole structure. As a result, pulses collapse onto the origin whereupon the configuration regains shape. No deflection angle appears.

To prove the claims we note that the solution reads neater with a skew transformation and a dilatation of coordinates defined by (48) and $z \equiv x^{\prime}-\beta y^{\prime}, \xi=\rho y^{\prime}$. Then dropping some irrelevant constants we have (see (42))

$$
\begin{gathered}
B \equiv B_{R}+i B_{I}=-\frac{f^{3}}{3}-\eta \frac{f^{2}}{2}+\omega y^{\prime}-C t, \\
B_{R}=-\frac{z^{3}}{3}+\xi^{2} z-\frac{\eta_{R}}{2}\left(z^{2}-\xi^{2}\right)+\eta_{I} \xi z+\omega_{R} \xi-t C_{R}, \\
B_{I}=\frac{\xi^{3}}{3}-\xi z^{2}-\frac{\eta_{I}}{2}\left(z^{2}-\xi^{2}\right)-\eta_{R} \xi z+\omega_{I} \xi-t C_{I} .
\end{gathered}
$$




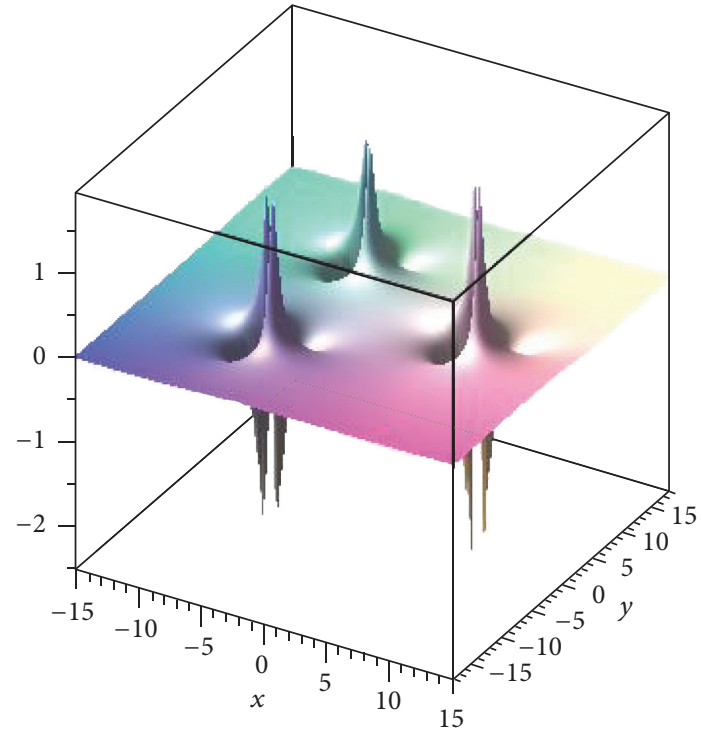

FIgUre 3: Homothetic solution at $t=-200$. The equilateral triangle is easily appreciated.

We simply consider here the case when $C=0$, namely, when the parameters are chosen to satisfy

$$
b v^{2}\left(1+\eta k_{1}\right)=i \eta k_{1}^{4} .
$$

The $t$-dependence of $\Delta$ drops out and hence the entire configuration $u(x, y, t)$ moves, with respect to the frame at rest, with constant velocity given by $\left(v,-b v^{2}\right)$. A plot of $u$ shows that $|u|^{2}-1$ decreases to zero away from three regions. Thus, the configuration behaves like a multipeaked solution of solitonic nature.

\section{Appendix}

\section{Mittag-Leffler Expansions of Eigenfunctions}

Recall that we assume that higher-order poles are the only singularities of $\mu(k)$; in particular, we exclude the possibility of having essential singularities and/or accumulation points of pole singularities in the finite plane. We study here the possibility of certain Mittag-Leffler expansions of eigenfunctions (see [35] for general ideas).

Theorem A.1. Let $\mu(k)$ be a meromorphic eigenfunction having poles at $S \equiv\left\{k_{n}\right\}_{n=1, \ldots, \infty}$, with canonical normalization. Suppose that

(1) the distance between eigenvalues is bounded below: $\delta \equiv$ $\sup _{n}\left|k_{n+1}-k_{n}\right|>0$,

(2) $\mu(k)$ is uniformly bounded away from eigenvalues.

Then, for $k \notin S, \mu(k)$ can be represented as

$$
\mu(k)=1+\sum_{k_{j} \in S} \mu_{\text {sing. }}^{\left.k_{j}\right)}(k) \equiv 1+\sum_{k_{j} \in S} \sum_{r=1}^{m_{j}} \frac{\phi_{j, r}}{\left(k-k_{j}\right)^{r}},
$$

where $m_{j}$ is the multiplicity of $k_{j}$ and $\mu_{\text {sing. }}^{k_{j}}(k)$ the pole divisor at $k_{j}$.

Reciprocally, if $\mu(k)$ is given by the formal series (A.1), condition (1) is satisfied and

$$
\begin{aligned}
\sum_{k_{j} \in S} \sum_{r=1}^{m_{j}}\left|\phi_{j, r}\right|<\infty, \\
\sum_{k_{j} \in S} \sum_{r=1}^{m_{j}}\left|\partial_{y} \phi_{j, r}\right|<\infty, \\
\sum_{k_{j} \in S} \sum_{r=1}^{m_{j}} k_{j}\left|\phi_{j, r}\right|<\infty, \quad M \equiv \sup _{n} m_{n}<\infty .
\end{aligned}
$$

Then $\mu$ is a meromorphic eigenfunction satisfying both condition (2) and also $\lim _{k \rightarrow \infty} \mu(k)=1$ along any curve $\mathscr{L}$ such that $\operatorname{Dist}(\mathscr{L}, S)>0$. Further,

$$
|u|^{2}=1+\sum_{k_{j} \in S} \partial_{y} \phi_{j, 1} \cdot
$$

Proof. The proposition is trivial if the number of pole singularities is finite so we shall assume an infinite number. Note then that (1) implies that they cannot accumulate anywhere on the finite plane. Thus, with no loss of generality we can assume that singularities pile up at infinity but not too quickly. We can order the eigenvalues so that $\left|k_{n}\right| \leq\left|k_{n+1}\right|$. Next, let set $S^{\prime} \subset S \equiv\left\{k_{n}\right\}_{n=1, \ldots, \infty}$ be the set obtained by choosing one eigenvalue from all those that have the same modulus. Let $a_{n} \equiv\left|k_{n}\right|, k_{n} \in S^{\prime}$. It follows that the sequence $\left\{a_{n}\right\}$ is strictly increasing: $a_{n}<a_{n+1}$ and satisfies $\lim _{n \rightarrow \infty} a_{n}=\infty$, $\delta \equiv \inf _{n}\left|a_{n}-a_{n+1}\right|>0$. Further, (2) implies that for all $l \in \mathbb{R}$ there exists $\epsilon(l)$ such that $|\mu(k)| \leq l$ for all $k_{n} \in S$ such that $\left|k-k_{n}\right| \geq \epsilon(l)$. It follows that there exists $C>0$ such that if $z \in \mathscr{R}_{n}$, the unit circle of radius $R_{n} \equiv\left(a_{n}+a_{n+1}\right) / 2$; then $|\mu(z)| \leq C$ uniformly in $n$ and $z: \sup _{n} \sup _{z \in \mathscr{R}_{n}}|\mu(z)| \leq C$. For fixed $k \notin S$ the function $\widetilde{\mu}(z) \equiv \mu(z) /(z-k)$ has poles with multiplicity $m_{n}$ at $k_{n}$ and a simple pole at $k$ with residue $\mu(k)$. At the former an easy calculation shows that $\operatorname{Res} \widetilde{\mu}(z)_{z=k_{n}}=$ $-\mu_{\text {sing. }}^{\left.k_{n}\right)}(k)$. Thus, Cauchy's theorem yields that

$$
\begin{aligned}
& \frac{1}{2 \pi} \int_{0}^{2 \pi} d \varphi \frac{\mu\left(R_{n} e^{i \varphi}\right)}{R_{n} e^{i \varphi}-k} R_{n} e^{i \varphi}=\frac{1}{2 \pi i} \int_{\mathscr{R}_{n}} \frac{\mu(z)}{z-k} d z \\
& \quad=\mu(k)-\sum_{j:\left|k_{j}\right|<R_{n}} \mu_{\text {sing. }}^{\left.k_{j}\right)}(k),
\end{aligned}
$$

where the sum extends to all eigenvalues $k_{j}$ satisfying $\left|k_{j}\right|<$ $R_{n}$.

Given such a fixed $k$ let $\epsilon$ be the minimum distance between $k$ and all $\mathscr{R}_{n}: \epsilon=\min _{n \in \mathbb{N}, z \in \mathscr{R}_{n}}|z-k|$ (note that $\epsilon>0$ ). We can bound the integrand, uniformly in $n$ as follows:

$$
\sup _{n}\left|\frac{\mu\left(R_{n} e^{i \varphi}\right)}{R_{n} e^{i \varphi}-k} R_{n} e^{i \varphi}\right| \leq \frac{C}{\epsilon} \in L_{1}([0,2 \pi], d \varphi),
$$


where we recall that $\left|\mu\left(R_{n} e^{i \varphi}\right)\right| \leq C$, say. Further, $\lim _{n \rightarrow \infty} R_{n}=$ $\infty, \lim _{R_{n} \rightarrow \infty} \mu\left(R_{n} e^{i \varphi}\right)=1$. By the theorem of dominated convergence we find that for fixed $k \notin S$

$$
\begin{aligned}
\lim _{R_{n} \rightarrow \infty} \int_{\mathscr{R}_{n}} \frac{\mu(z)}{z-k} d z & =i \int_{0}^{2 \pi} d \varphi \lim _{R_{n} \rightarrow \infty} \frac{\mu\left(R_{n} e^{i \varphi}\right)}{R_{n} e^{i \varphi}-k} R_{n} e^{i \varphi} \\
& =2 \pi i .
\end{aligned}
$$

Sending $n \rightarrow \infty$ the result follows.

Reciprocally, if $\mu(k)$ is given by the formal series (A.1) and condition (1) is satisfied (with $\delta \leq 1$, say), we see that $\mu(k)$ exists and, by formally commuting the limit $k \rightarrow \infty$ with the sum, find that $\mu(k)$ tends to 1 at infinity. The interchange of limit and sum is validated by noting that $\operatorname{if} \operatorname{Dist}(\mathscr{L}, S) \geq \epsilon>0$,

$$
\sum_{k_{j} \in S} \sum_{r=1}^{m_{j}} \frac{\left|\phi_{j, r}\right|}{\left|k-k_{j}\right|^{r}} \leq \frac{1}{\epsilon^{M}} \sum_{k_{j} \in S} \sum_{r=1}^{m_{j}}\left|\phi_{j, r}\right|<\infty .
$$

To prove that the potential is bounded, we use (10):

$$
\begin{aligned}
\mu^{1)} & =\lim _{k \rightarrow \infty} k \sum_{k_{j} \in S} \sum_{r=1}^{m_{j}} \frac{\phi_{j, r}}{\left(k-k_{j}\right)^{r}} \\
& =\sum_{k_{j} \in S} \sum_{r=1}^{m_{j}} \lim _{k \rightarrow \infty} \frac{k}{\left(k-k_{j}\right)^{r}} \phi_{j, r}=\sum_{k_{j} \in S} \sum_{r=1}^{m_{j}} \phi_{j, r},
\end{aligned}
$$

where we have taken the limit under the sum. To validate this note that if $\operatorname{Dist}(\mathscr{L}, S) \geq \epsilon>0$, the modulus-maximum principle yields the estimate uniform on $k$ :

$$
\begin{aligned}
\frac{\left|k \phi_{r, j}\right|}{\left|k-k_{j}\right|^{r}} & \leq \frac{1}{\epsilon^{r}}\left(\epsilon+\left|k_{j}\right|\right)\left|\phi_{j, r}\right| \\
\left|\frac{k \phi_{j, r}}{\left(k-k_{j}\right)^{r}}\right| & \leq \sum_{k_{j} \in S} \sum_{r=1}^{m_{j}} \frac{1}{\epsilon^{r}}\left|\epsilon+k_{j}\right|\left|\phi_{j, r}\right| \\
& \leq \frac{1}{\epsilon^{M}} \sum_{k_{j} \in S} \sum_{r=1}^{m_{j}}\left(|\epsilon|+\left|k_{j}\right|\right)\left|\phi_{j, r}\right|<\infty .
\end{aligned}
$$

Thus, if the conditions hold we are guaranteed that $\mu(k)$ has an asymptotic expansion $\mu(k)=1+\mu^{1)}+O(1 / k)$, where $\mu^{1)}$ is given by (A.8). Operating with $\partial_{y}$ and since (A.2) validates differentiation under the sum we arrive to Formula (A.3).

The above result can be sharpened when eigenvalues are disposed along a curve.

Proposition A.2. Suppose that the set $S$ is contained in a given line $\mathscr{H} \equiv\left\{k \in \mathbb{C}: k=r e^{i \varphi}, 0<r<\infty\right\}$ for some $\varphi$ and satisfies condition (1). Suppose that the formal series (A.1) $\equiv$ $\mu(k)$ satisfies all conditions (A.2) but the third; that is, we drop the requirement $\sum_{k_{j}} \sum_{r=1}^{m_{j}} k_{j}\left|\phi_{j, r}\right|<\infty$. For any $\epsilon>0$ let $D_{\epsilon} \supset$ $\mathscr{H}$ be the cone of complex numbers whose phase $\vartheta$ satisfies $\varphi-$ $\epsilon<\vartheta<\varphi+\epsilon$. Then, $\mu(k)$ has, along any curve contained in
$\mathbb{C}-D_{\epsilon}$, an asymptotic expansion $\mu(k)=1+\mu^{1)}+O(1 / k)$, where $\mu^{1)}$ is given by (A.8). Hence, it is associated with a nonsingular potential u given again by (A.3).

Proof. We have the uniform bound:

$$
\sup _{k \in \mathbb{C}-D_{\epsilon}} \frac{\left|k \phi_{j, r}\right|}{\left|k-k_{j}\right|^{r}}=\sup _{k \in \partial D_{\epsilon}} \frac{\left|k \phi_{j, r}\right|}{\left|k-k_{j}\right|^{r}} \leq|\sin \epsilon|^{-r}\left|\phi_{j, r}\right|,
$$

where we use first the modulus-maximum principle for analytic functions and then, with no loss of generality, suppose $|k| \geq 1$. For given $k_{j}$ the sup is attained at the normal projection of $k_{j}$ onto the ray $\partial D_{\epsilon}$ (note that this estimate can be sharpened but with no further gain). It implies that, along any curve contained in $\mathbb{C}-D_{\epsilon}$,

$$
\mu^{1)}=\lim _{k \rightarrow \infty} k \sum_{k_{j} \in S} \sum_{r=1}^{m_{j}} \frac{\phi_{j, r}}{\left(k-k_{j}\right)^{r}}=\sum_{k_{j} \in S} \sum_{r=1}^{m_{j}} \phi_{j, r} .
$$

Proposition A.3. Suppose that $\mu(k)$ is given by the formal series (A.1) and conditions (1) and (2) are satisfied. Suppose that the set $S$ is contained in a given line $\mathscr{H} \equiv\{k \in \mathbb{C}: k=$ $\left.r e^{i \varphi}, 0<r<\infty\right\}$ for some $\varphi$. With no loss of generality order eigenvalues are such that $\left|k_{n}\right| \leq\left|k_{n+1}\right|$. Further suppose that $\sum_{n=1}^{\infty}\left(1 /\left|k_{n}\right|\right)<\infty$. Then the formal series (A.1) is convergent to $\mu(k)$ having canonical normalization and is associated with a nonsingular potential $u$.

A proof of this assertion follows by noting that (24) imply that for long $n, \phi_{n}$ can be expanded as

$$
\phi_{n}=\frac{1}{x+2 i k_{n} t-y / k_{n}^{2}}+O\left(\frac{1}{k_{n}^{2}}\right) .
$$

For $t \neq 0$ both conditions of (A.2) are satisfied. This implies the claim.

\section{Competing Interests}

The authors declare herewith that there is no conflict of interests regarding the publication of this article. Our professional judgment concerning the validity of research is not influenced by a secondary interest such as financial gain.

\section{Acknowledgments}

The authors acknowledge support from the Spanish Ministerio de Economía y Competitividad under Contracts MTM2012-38445, MAT2013-46308, and Junta de Castilla y Leon SA226U13.

\section{References}

[1] A. S. Fokas, "On the simplest integrable equation in 2+1," Inverse Problems, vol. 10, no. 2, pp. L19-L22, 1994.

[2] E. I. Shul'man, "On the integrability of equations of DaveyStewartson type," Theoretical and Mathematical Physics, vol. 56, no. 1, pp. 720-724, 1983. 
[3] M. Boiti, J. J. P. Leon, and F. Pempinelli, "Spectral transform for a two spatial dimension extension of the dispersive long wave equation," Inverse Problems, vol. 3, no. 3, pp. 371-387, 1987.

[4] M. Boiti, F. Pempinelli, and A. Pogrebkov, "Solutions of the KPI equation with smooth initial data," Inverse Problems, vol. 10, no. 3, pp. 505-519, 1994.

[5] J. Villarroel and J. Prada, "Considerations on conserved quantities and boundary conditions of the 2+1-dimensional nonlinear Schrödinger equation," Physica D: Nonlinear Phenomena, vol. 300, pp. 15-25, 2015.

[6] S. Chakravarty, L. Kent, and T. Newmann, "Some reductions of the self-dual Yang-Mills equations to integrable systems in $2+1$ dimensions," Journal of Mathematical Physics, vol. 36, no. 2, pp. 763-772, 1995.

[7] A. S. Fokas and M. J. Ablowitz, "On the inverse scattering of the time dependent schrödinger equation and the associated KPI equation," Studies in Applied Mathematics, vol. 69, no. 3, pp. 211$228,1983$.

[8] A. S. Fokas and M. J. Ablowitz, "The inverse scattering transform for multidimensional $(2+1)$ problems," in Nonlinear Phenomena, K. B. Wolf, Ed., vol. 189 of Lecture Notes in Physics, pp. 137-183, Springer, Berlin, Germany, 1983.

[9] M. J. Ablowitz and A. Fokas, "Comments on the inverse scattering transform and related nonlinear evolution equations," in Nonlinear Phenomena, vol. 189 of Lecture Notes in Physics, pp. 4-23, Springer, Berlin, Germany, 1983.

[10] M. J. Ablowitz and P. A. Clarkson, Solitons, Non-linear Evolution Equations \& Inverse Scattering, Cambridge University Press, Cambridge, UK, 1992.

[11] V. A. Arkadiev, A. K. Pogrebkov, and M. C. Polivanov, "Inverse scattering transform method and soliton solutions for DaveyStewartson II equation," Physica D: Nonlinear Phenomena, vol. 36, no. 1-2, pp. 189-197, 1989.

[12] V. Lipovski and V. Shirokov, " $2+1$ Toda chain. I. Inverse scattering method," Theoretical and Mathematical Physics, vol. 75, no. 3, pp. 555-566, 1988.

[13] J. Villarroel and M. J. Ablowitz, "On the inverse scattering transform of the $2+1$ Toda equation," Physica D: Nonlinear Phenomena, vol. 65, no. 1-2, pp. 48-70, 1993.

[14] J. Villarroel and M. J. Ablowitz, "On the initial value problem for the KPII equation with data that do not decay along a line," Nonlinearity, vol. 17, no. 5, pp. 1843-1866, 2004.

[15] V. M. Galkin, D. E. Pelinovsky, and Y. A. Stepanyants, "The structure of the rational solutions to the Boussinesq equation," Physica D: Nonlinear Phenomena, vol. 80, no. 3, pp. 246-255, 1995.

[16] K. A. Gorshov, D. E. Pelinovskii, and Yu. A. Stepanyants, "Normal and anomalous scattering, formation and decay of bound states of two-dimensional solitons described by the KadomtsevPetviashvili equation," Journal of Experimental and Theoretical Physics, vol. 77, no. 2, pp. 237-245, 1993.

[17] M. J. Ablowitz and J. Villarroel, "Solutions to the time dependent Schrödinger and the Kadomtsev-Petviashvili equations," Physical Review Letters, vol. 78, no. 4, pp. 570-573, 1997.

[18] J. Villarroel and M. J. Ablowitz, "On the discrete spectrum of the nonstationary Schrödinger equation and multipole lumps of the Kadomtsev-Petviashvili I equation," Communications in Mathematical Physics, vol. 207, no. 1, pp. 1-42, 1999.

[19] M. J. Ablowitz and J. Villarroel, New Trends in Integrability, Kluwer Academic, New York, NY, USA, 2004.
[20] J. Villarroel and M. J. Ablowitz, "On the discrete spectrum of systems in the plane and the davey-stewartson II equation," SIAM Journal on Mathematical Analysis, vol. 34, no. 6, pp. 12531278, 2003.

[21] G. Biondini and S. Chakravarty, "Soliton solutions of the Kadomtsev-Petviashvili II equation," Journal of Mathematical Physics, vol. 47, no. 3, Article ID 033514, 2006.

[22] S. Chakravarty and Y. Kodama, "Soliton solutions of the KP equation and application to shallow water waves," Studies in Applied Mathematics, vol. 123, no. 1, pp. 83-151, 2009.

[23] M. Boiti, F. Pempinelli, A. K. Pogrebkov, and B. Prinari, "Inverse scattering theory of the heat equation for a perturbed onesoliton potential," Journal of Mathematical Physics, vol. 43, no. 2, pp. 1044-1062, 2002.

[24] Q. P. Liu and M. Mañas, "Vectorial Darboux transformations for the Kadomtsev-Petviashvili hierarchy," Journal of Nonlinear Science, vol. 9, no. 2, pp. 213-232, 1999.

[25] M. J. Ablowitz, S. Chakravarty, A. D. Trubatch, and J. Villarroel, "A novel class of solutions of the non-stationary Schrodinger and the Kadomtsev-Petviashvili I equations," Physics Letters, Section A: General, Atomic and Solid State Physics, vol. 267, no. 2-3, pp. 132-146, 2000.

[26] M. Mañas and P. M. Santini, "Solutions of the Davey-Stewartson II equation with arbitrary rational localization and nontrivial interaction," Physics Letters. A, vol. 227, no. 5-6, pp. 325-334, 1997.

[27] A. S. Fokas, D. E. Pelinovsky, and C. Sulem, "Interaction of lumps with a line soliton for the DSII equation," Physica D: Nonlinear Phenomena, vol. 152-153, pp. 189-198, 2001.

[28] R. Radha and M. Lakshmanan, "Exotic coherent structures in the $(2+1)$-dimensional long dispersive wave equation," Journal of Mathematical Physics, vol. 38, no. 1, pp. 292-299, 1997.

[29] K. Porsezian, "Painlevé analysis of new higher-dimensional soliton equation," Journal of Mathematical Physics, vol. 38, no. 9, pp. 4675-4679, 1997.

[30] C.-L. Bai, H. Zhao, and X.-Y. Wang, "The structures and interactions of solitary waves in the $(2+1)$-dimensional nonlinear Schrödinger equation," Nonlinearity, vol. 19, no. 8, pp. 1697-1712, 2006.

[31] P. G. Estévez, "Darboux transformation and solutions for an equation in $2+1$ dimensions," Journal of Mathematical Physics, vol. 40, no. 3, pp. 1406-1419, 1999.

[32] J. Villarroel, J. Prada, and P. G. Estévez, "Dynamics of lump solutions in a $2+1$ NLS equation," Studies in Applied Mathematics, vol. 122, no. 4, pp. 395-410, 2009.

[33] J. M. Cerveró and P. G. Estévez, "Miura transformation between two non-linear equations in $2+1$ dimensions," Journal of Mathematical Physics, vol. 39, no. 5, pp. 2800-2807, 1998.

[34] P. G. Estévez, J. Prada, and J. Villarroel, "On an algorithmic construction of lump solutions in a $2+1$ integrable equation," Journal of Physics A: Mathematical and Theoretical, vol. 40, no. 26, pp. 7213-7231, 2007.

[35] M. J. Ablowitz and A. S. Fokas, Complex Variables: Introduction and Applications, Cambridge University Press, Cambridge, UK, 1997. 


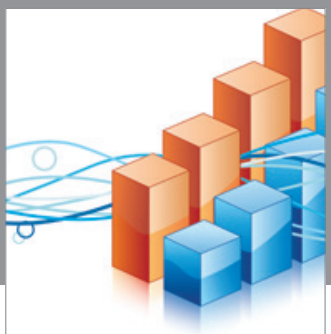

Advances in

Operations Research

vatem alat4



\section{The Scientific} World Journal
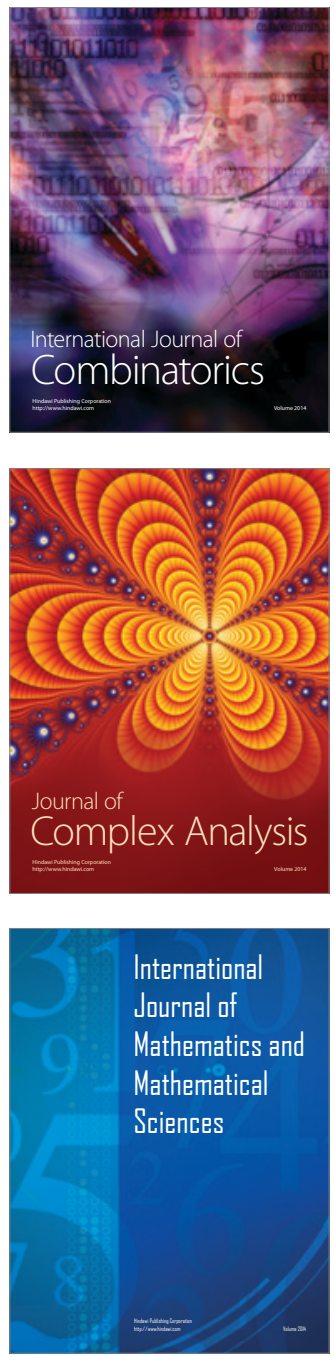
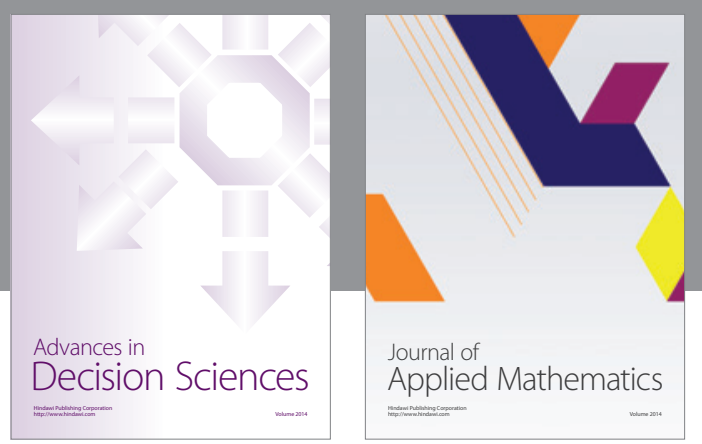

Algebra

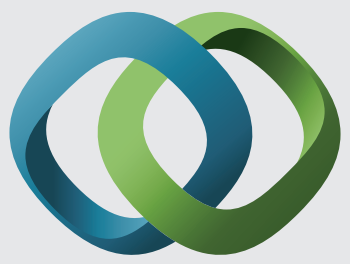

\section{Hindawi}

Submit your manuscripts at

http://www.hindawi.com
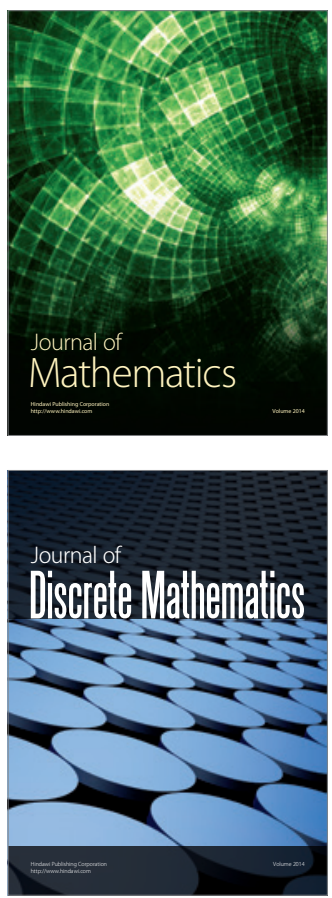

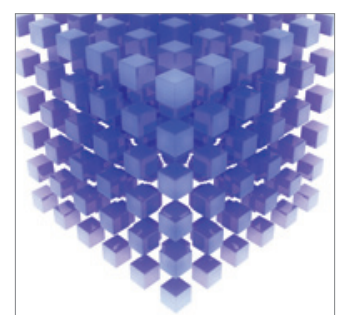

Mathematical Problems in Engineering
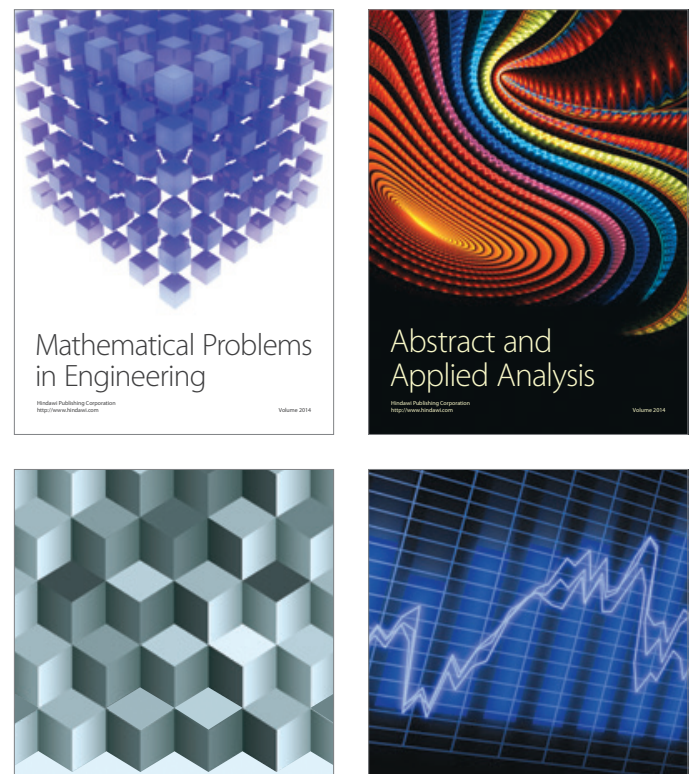

Journal of

Function Spaces

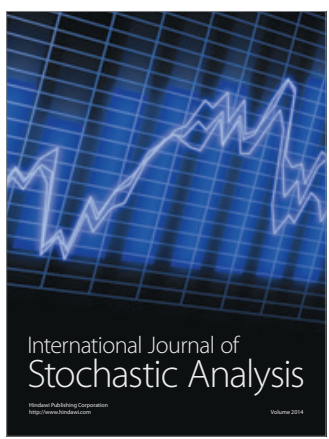

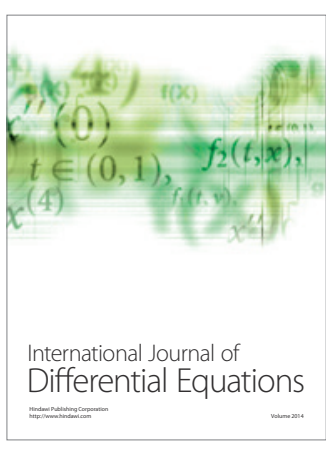
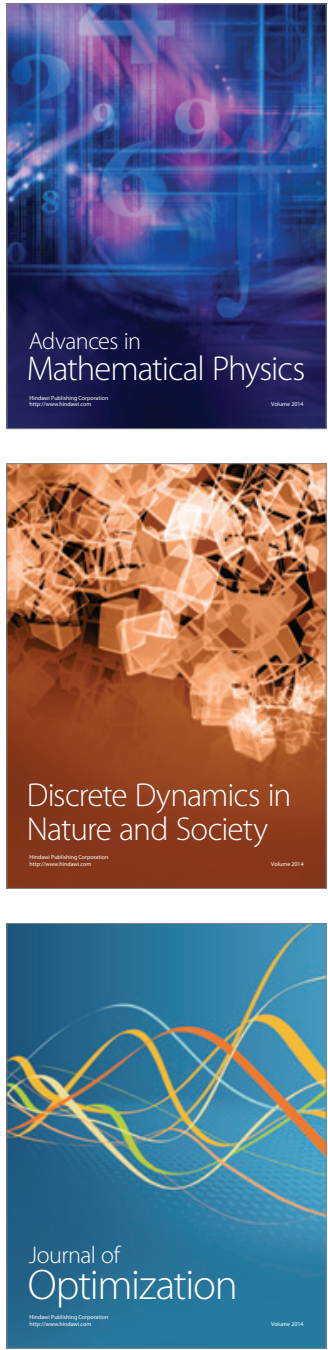